\title{
Shoulder Support Device
}

National Cancer Institute

\section{Source}

National Cancer Institute. Shoulder Support Device. NCI Thesaurus. Code C50305.

A device designed to support an object as it sits on the shoulder or an orthopedic device designed for rehabilitation after a shoulder injury. 